\title{
Response to comments on "Short Note: Extending simplified high-degree synthesis methods to second latitudinal derivatives of geopotential"
}

\author{
S. A. Holmes • W. E. Featherstone
}

Received: 29 May 2014 / Accepted: 2 June 2014 / Published online: 28 June 2014

(C) Springer-Verlag Berlin Heidelberg 2014

We would like to thank Dr. Crouse for taking sufficient interest in our paper so as to correctly and independently detect the typographical error in the initial (un-numbered) equation of Holmes and Featherstone (2002). We also thank him for agreeing to incorporate our own additional corrections into his comment, these being the superfluous modulus symbols that encompass many of the Legendre terms. Our records show these symbols are an unexpected contribution during the typesetting process. We were surprised to see these modulus symbols in the published manuscript, and are now pleased to see them finally identified and dealt with.

Importantly, we note that Dr. Crouse's comment makes no mention as to whether the original and incorrectly printed equation was used to generate the results in Section 3 of Holmes and Featherstone (2002). If so, this would invalidate all the results and conclusions of our paper, so we are puzzled as to why this issue has been ignored in this comment. Of course, verifying the computations in Section 3 requires substantially more effort than simply identifying typographical errors, especially since the majority were identified and provided by us. We hereby confirm that the correct equations were used in all computations leading towards the results and conclusions of Holmes and Featherstone (2002).

S. A. Holmes

SGT Inc., 7701 Greenbelt Road, Suite 400,

Greenbelt, MD 20770, USA

e-mail: sholmes@sgt-inc.com

W. E. Featherstone $(\varangle)$

Western Australian Centre for Geodesy and The Institute for Geoscience Research, Curtin University of Technology, GPO Box U1987, Perth, WA 6845, Australia

e-mail: w.featherstone@curtin.edu.au

\section{References}

Holmes SA, Featherstone WE (2002) Short Note: Extending simplified high-degree synthesis methods to second latitude derivatives of geopotential. J Geodesy 76(8):447-450. doi:10.1007/ s00190-002-0268-3 